\title{
Mapping superintegrable quantum mechanics to resonant spacetimes
}

\author{
Oleg Evnin, ${ }^{1,2, *}$ Hovhannes Demirchian, ${ }^{3, \dagger}$ and Armen Nersessian ${ }^{4,5, \$}$ \\ ${ }^{1}$ Department of Physics, Faculty of Science, Chulalongkorn University, Bangkok 10330, Thailand \\ ${ }^{2}$ Theoretische Natuurkunde, Vrije Universiteit Brussel (VUB) and The International Solvay Institutes, \\ Brussels 1050, Belgium \\ ${ }^{3}$ Ambartsumian Byurakan Astrophysical Observatory, Byurakan 0213, Armenia \\ ${ }^{4}$ Yerevan Physics Institute, 2 Alikhanyan Brothers Street, Yerevan 0036, Armenia \\ ${ }^{5}$ Bogoliubov Laboratory of Theoretical Physics, Joint Institute for Nuclear Research, Dubna 141980, Russia
}

(Received 18 November 2017; published 24 January 2018)

\begin{abstract}
We describe a procedure naturally associating relativistic Klein-Gordon equations in static curved spacetimes to nonrelativistic quantum motion on curved spaces in the presence of a potential. Our procedure is particularly attractive in application to (typically, superintegrable) problems whose energy spectrum is given by a quadratic function of the energy level number, since for such systems the spacetimes one obtains possess evenly spaced, resonant spectra of frequencies for scalar fields of a certain mass. This construction emerges as a generalization of the previously studied correspondence between the Higgs oscillator and anti-de Sitter spacetime, which has been useful for both understanding weakly nonlinear dynamics in anti-de Sitter spacetime and algebras of conserved quantities of the Higgs oscillator. Our conversion procedure ("Klein-Gordonization") reduces to a nonlinear elliptic equation closely reminiscent of the one emerging in relation to the celebrated Yamabe problem of differential geometry. As an illustration, we explicitly demonstrate how to apply this procedure to superintegrable Rosochatius systems, resulting in a large family of spacetimes with resonant spectra for massless wave equations.
\end{abstract}

DOI: 10.1103/PhysRevD.97.025014

\section{INTRODUCTION}

Geometrization of dynamics is a recurrent theme in theoretical physics. While it has underlain such fundamental developments as the creation of general relativity and search for unified theories of interactions, it also has a more modest (but often fruitful) aspect of reformulating conventional, well-established theories in more geometrical terms, in the hope of elucidating their structure. One particular approach of the latter type is the Jacobi metric (for a contemporary treatment, see [1-3]). This energy-dependent metric simply encodes as its geodesics the classical orbits of a nonrelativistic mechanical particle on a manifold moving in a potential.

The geometrization program we propose here can be seen as a quantum counterpart of the Jacobi metric. To a nonrelativistic quantum particle on a manifold moving in a potential, we associate a relativistic Klein-Gordon equation in a static spacetime of one dimension higher. Since the

\footnotetext{
*oleg.evnin@gmail.com

†demhov@yahoo.com

*arnerses@ysu.am
}

Published by the American Physical Society under the terms of the Creative Commons Attribution 4.0 International license. Further distribution of this work must maintain attribution to the author(s) and the published article's title, journal citation, and DOI. Funded by SCOAP.
Klein-Gordon equation can be seen as a sort of quantization of geodesics (and reduces to the geodesic equation in the eikonal approximation), this provides a quantized version of the correspondence between particle motion on a manifold in the presence of a potential and purely geometric geodesic motion in the corresponding spacetime. Executing our geometrization algorithm in general reduces to a nonlinear elliptic equation closely reminiscent of the one emerging in relation to the Yamabe problem and its generalizations known as prescribed scalar curvature problems [4-6], and thus connects to extensive literature and interesting questions in differential geometry. (The Yamabe problem refers to constructing a conformal transformation of the given metric on a manifold that makes the Ricci scalar of the conformally transformed metric constant.)

While the correspondence we build may, in principle, operate on any system, we are primarily motivated by its application to a very special class of quantum systems whose energy is a quadratic function of the energy level number. Such systems are exemplified by the one-dimensional Pöschl-Teller potential, and in higher dimensions they are typically superintegrable. In fact, our construction has been developed precisely as a generalization of the correspondence [7-9] between the Higgs oscillator [10,11], a particularly simple superintegrable system with a quadratic spectrum, and Klein-Gordon equations on the anti-de Sitter (AdS) spacetime, the maximally symmetric spacetime of 
constant negative curvature. This correspondence has emerged in the context of studying selection rules [12-14] in the nonlinear perturbation theory targeting the AdS stability problem $[15,16]$. The correspondence has been useful for both elucidating the structure of AdS perturbation theory [8] and for resolving the old problem of constructing explicit hidden symmetry generators for the Higgs oscillator [9].

The reason for our emphasis on systems with quadratic spectra is that, in application to such systems, our geometrization program generates Klein-Gordon equations whose frequency spectra are linear in the frequency level number, and hence the spectrum is highly resonant (the difference of any two frequencies is integer in appropriate units). It is well known that in the context of weakly nonlinear dynamics, highly resonant spectra have a dramatic impact, as they allow arbitrarily small nonlinear perturbations to produce arbitrarily large effects over long times. This feature has been crucial in the extensive investigations of the AdS stability problem in the literature (for a brief review and references, see [16]). The main practical target of our geometrization program thus appears twofold.

(i) To provide geometric counterparts for quantum systems with quadratic spectra (the resulting Klein-Gordon equation is set up on a highly special spacetime with a resonant spectrum of frequencies and the geometric properties of this spacetime are likely to yield insights into the algebraic properties of the original quantum system, including its high degree of degeneracy and hidden symmetries).

(ii) To generate, starting from known quantum systems with quadratic spectra, highly resonant spacetimes (weakly nonlinear dynamics in such spacetimes is likely to be very sophisticated, sharing the features of the extensively explored weakly nonlinear dynamics of AdS spacetime).

The plan of the paper is as follows. In Sec. II, we formulate our general geometrization procedure and describe how it simplifies for the case of zero mass in the Klein-Gordon equation one is aiming to construct. In Sec. III, we describe how the previously known correspondence [7-9] between the Higgs oscillator and AdS fits in our general framework. In Sec. IV, we analyze the superintegrable Rosochatius system, which generalizes the Higgs oscillator, and generate a large family of spacetimes perfectly resonant with respect to the massless wave equation. We conclude with a review of the current state of our formalism and open problems.

\section{KLEIN-GORDON}

\section{A. General formulation of the Klein-Gordonization procedure}

Consider a quantum system with the Hamiltonian

$$
H=-\Delta_{\gamma}+V(x),
$$

where $\Delta_{\gamma} \equiv \gamma^{-1 / 2} \partial_{i}\left(\gamma^{1 / 2} \gamma^{i j} \partial_{j}\right)$ is the Laplacian on a $d$-dimensional manifold parametrized with $x^{i}$ and endowed with the metric $\gamma_{i j}$. We are particularly interested in systems whose energy spectrum consists of (in general, degenerate) energy levels labeled by the level number $N=0,1, \ldots$, and the energy is a quadratic function of the level number,

$$
E_{N}=A(N+B)^{2}-C .
$$

Such spectra are indeed observed in a number of interesting systems, typically involving superintegrability, for example the following:

(i) The Higgs oscillator [10,11], which is a particle on a $d$-sphere moving in a potential varying as the inverse cosine squared of the polar angle.

(ii) The superintegrable version $[17,18]$ of the Rosochatius system on a $d$-sphere $[19,20]$, which is the most direct generalization of the Higgs oscillator.

(iii) The quantum angular Calogero-Moser model [21].

(iv) The (spherical) Calogero-Higgs system [22,23].

We additionally mention the following two completely elementary systems, which give a particularly simple realization of the quadratic spectrum (2):

(i) A particle in one dimension in an infinite rectangular potential well.

(ii) The trigonometric Pöschl-Teller system [24].

We associate to any system of the form (1) a KleinGordon equation in a certain static $(d+1)$-dimensional spacetime. We introduce a scalar field $\tilde{\phi}(t, x)$ satisfying

$$
-\partial_{t}^{2} \tilde{\phi}=\left(-\Delta_{\gamma}+V(x)+C\right) \tilde{\phi} .
$$

In the above expression, $C$ can, in principle, be an arbitrary constant, but our main focus is on systems with energy spectrum of the form (2) and $C$ read off from (2). One can equivalently write (3) as

$$
\square_{\tilde{g}} \tilde{\phi}-(C+V(x)) \tilde{\phi}=0,
$$

where $\square_{\tilde{g}}$ is the D'Alembertian of the metric

$$
\tilde{g}_{\mu \nu} d x^{\mu} d x^{\nu}=-d t^{2}+\gamma_{i j} d x^{i} d x^{j},
$$

with $x^{\mu}=\left(t, x^{i}\right)$. By construction, if one implements separation of variables in (4) in the form

$$
\tilde{\phi}=e^{i w t} \Psi(x),
$$

one recovers the original Schrödinger equation as $H \Psi=\left(w^{2}-C\right) \Psi$. This guarantees that the mode functions of (4) are directly related to the energy eigenstates of the original quantum-mechanical problem. Note that, if one focuses on systems with energy spectra of the form (2), by construction, separation of variables in (3) leads to eigenmodes with linearly spaced frequencies, 


$$
w_{N}=\sqrt{A}(N+B)
$$

In this case, after conversion to the Klein-Gordon form, which we undertake below, the resulting spacetime possesses a resonant spectrum of frequencies.

Equation (3) is not of a Klein-Gordon form, but we can try to put it in this form by applying a conformal rescaling to $\tilde{g}$ and $\tilde{\phi}$,

$$
\tilde{g}_{\mu \nu}=\Omega^{2} g_{\mu \nu}, \quad \tilde{\phi}=\Omega^{\frac{1-d}{2}} \phi .
$$

One thus gets (relevant conformal transformation formulas can be retrieved, e.g., from [25])

$$
\begin{gathered}
\square_{g} \phi-\left[(C+V(x)) \Omega^{2}+\frac{d-1}{2} \frac{\square_{g} \Omega}{\Omega}\right. \\
\left.+\frac{(d-1)(d-3)}{4} \frac{g^{\mu \nu} \partial_{\mu} \Omega \partial_{\nu} \Omega}{\Omega^{2}}\right] \phi=0 .
\end{gathered}
$$

If the expression in the square brackets can be made constant by a suitable choice of $\Omega$, we get a KleinGordon equation in a spacetime with the metric $g_{\mu \nu}$. We thus need to solve the equation

$$
\begin{aligned}
(C & +V(x)) \Omega^{2}+\frac{d-1}{2} \frac{\square_{g} \Omega}{\Omega} \\
& +\frac{(d-1)(d-3)}{4} \frac{g^{\mu \nu} \partial_{\mu} \Omega \partial_{\nu} \Omega}{\Omega^{2}}=m^{2}
\end{aligned}
$$

It is wiser to rewrite this equation through the metric $\tilde{g}$, which is already known and given by (5),

$$
\frac{d-1}{2} \Omega \square_{\tilde{g}} \Omega-\frac{d^{2}-1}{4} \tilde{g}^{\mu \nu} \partial_{\mu} \Omega \partial_{\nu} \Omega+(C+V(x)) \Omega^{2}=m^{2} .
$$

Since neither $V(x)$ nor $\tilde{g}_{\mu \nu}$ depend on $t$, one can assume that $\Omega$ is a function of $x^{i}$ as well. Hence,

$$
\frac{d-1}{2} \Omega \Delta_{\gamma} \Omega-\frac{d^{2}-1}{4} \gamma^{i j} \partial_{i} \Omega \partial_{j} \Omega+(C+V(x)) \Omega^{2}=m^{2} .
$$

Note that (10) is closely reminiscent of the equation emerging from the following purely geometrical problem: Consider a metric $g_{\mu \nu}$ whose Ricci scalar is $R(x)$. Is it possible to find $\Omega$ such that the Ricci scalar corresponding to $\tilde{g}_{\mu \nu}=\Omega^{2} g_{\mu \nu}$ has a given form $\tilde{R}(x)$ ? Indeed, from the standard formulas for the change of the Ricci scalar under conformal transformations, see, e.g., (3.4) of [25], one gets
$\Omega^{2} \tilde{R}(x)=R(x)+2 d \frac{\square_{g} \Omega}{\Omega}+d(d-3) \frac{g^{\mu \nu} \partial_{\mu} \Omega \partial_{\nu} \Omega}{\Omega^{2}}$.

Algebraically, this has the same structure as (10).

Equations of the form (13) for simple specific choices of $g$ and $\tilde{R}$ have been studied in mathematical literature as various realizations of the "prescribed scalar curvature" problem [6]. The substitution

$$
\Omega=\omega^{-\frac{2}{d-1}}
$$

reduces (12) to the following compact form,

$$
-\Delta_{\gamma} \omega+(C+V(x)) \omega=m^{2} \omega^{\frac{d+3}{d-1}}
$$

closely reminiscent of the equation arising in relation to the Yamabe problem [4-6]. (Note that the specific power of $\omega$ appearing on the right-hand side of this equation is different from the standard Yamabe problem. This is because we are performing a conformal transformation in a spacetime of one dimension higher, rather than in the original space.) Once (15) has been solved, the spacetime providing geometrization of the original problem (1) can be written explicitly as

$$
g_{\mu \nu} d x^{\mu} d x^{\nu}=\omega^{\frac{4}{d-1}}\left(-d t^{2}+\gamma_{i j} d x^{i} d x^{j}\right)
$$

Equation (12) dramatically simplifies in one spatial dimension $(d=1)$, where all the derivative terms drop out, leaving $\Omega \sqrt{C+V(x)}=m$. Thus, for the particle in an infinite rectangular potential well, Klein-Gordonization gives a massless wave equation on a slice of Minkowski space between two mirrors, while for the Pöschl-Teller system, one immediately obtains a two-dimensional spacetime metric reminiscent of anti-de Sitter spacetime $\mathrm{AdS}_{2}$. This latter result displays some parallels to the considerations of [26] (focusing in the hyperbolic Pöschl-Teller system).

As we already briefly remarked, the above geometrization procedure can be applied to any Hamiltonian of the form (1) and any $C$, irrespectively of the form of the spectrum. However, it is precisely for the spectrum and $C$ given by (2) that the resulting spacetime possesses the remarkable property of being highly resonant (and one may expect that its geometric properties will give a more transparent underlying pictures of the algebraic structures of the original quantum-mechanical problem, as happens for the Higgs oscillator). We therefore focus on the application of our geometrization procedure to such systems with quadratic energy spectra.

\section{B. The massless case}

Equation (15) is a nonlinear elliptic equation and in general difficult to solve. Extensive existence results are established for an algebraically similar equation arising in relation to the Yamabe problem; hence one may hope that 
some level of understanding of solutions to (15) in full generality may also be attained in the future. We do not pursue such systematic analysis here, however.

Driven by practical goals of constructing resonant spacetimes and geometrizing concrete superintegrable systems, we point out that (15) becomes linear and dramatically simplifies if one assumes $m^{2}=0$. Hence, converting a given quantum-mechanical problem to a massless wave equation is considerably simpler than for general values of the mass.

We note that, if $m^{2}=0$, Eq. (15) looks identical to the Schrödinger equation corresponding to the Hamiltonian (1), with energy eigenvalue $-C$,

$$
-\Delta_{\gamma} \omega+V(x) \omega=-C \omega .
$$

(Normalizable eigenstates of this energy do not generically exist, but $\omega$ does not have to satisfy the same normalizability conditions as standard wave functions; hence this should not be a problem.) Since quadratic spectra (2) are seen to arise from highly structured, typically superintegrable, systems, one may naturally expect that (17) is amenable to analytic treatment.

There is one further assumption one might make that immediately yields solutions of (17) from known solutions of the original quantum-mechanical problem (1). Namely, imagine one has a $K$-parameter family of Hamiltonians (1) with quadratic spectra (2). In this case, $A, B$, and $C$ are functions of the $K$ parameters defining our family of Hamiltonians. One may impose

$$
B=0,
$$

which generically yields a $(K-1)$-parameter subfamily of quantum systems with quadratic spectra. Within this subfamily, the ground state $\Psi_{0}$ has the energy $-C$, i.e., $H \Psi_{0}=-C \Psi_{0}$. Hence, $\omega$ satisfying (17) can be chosen as the vacuum state of $H$,

$$
\omega=\Psi_{0} .
$$

We make use of this construction below, as it allows for a straightforward application of our methodology to known exactly solvable systems. (In some cases, it is geometrically advantageous to use the non-normalizable counterpart of $\Psi_{0}$ with the same energy eigenvalue to define $\omega$. Such nonnormalizable states should also be easy to construct for exactly solvable systems with quadratic spectra. We see an explicit realization of this scenario in our subsequent treatment of the superintegrable Rosochatius problem.)

As a variation of the above special case, one could force $B$ of (2) to be equal to a negative integer and $\omega$ to be equal to an excited state wave function. This, however, introduces singularities in the conformally rescaled spacetime (16) at the location of zeros of the excited state wave functions. While one could still try to pursue this scenario by imposing appropriate constraints on the wave equation solution at the singular locus, we concentrate below on the most straightforward formulation (19) utilizing the ground state wave function, where the conformal factor is nonvanishing and no such subtleties arise.

\section{HIGGS}

Before proceeding with novel derivations we demonstrate how the case of the Higgs oscillator, which has motivated our general construction, fits into our present framework. We are essentially just reviewing the derivations in [7-9].

The Higgs oscillator is a particle on a sphere moving in a specific centrally symmetric potential (which we specify below). It is remarkable for being one of only three centrally symmetric maximally superintegrable systems on a sphere (together with free motion and the spherical Coulomb potential). A practical manifestation of superintegrability is that all of its classical trajectories are closed. The quantum version of this system attracted considerable attention after it was reintroduced in a different guise and solved in [27]. The observed high degeneracy of energy levels of this system prompted investigation of its hidden symmetries in $[10,11]$, which resulted in identification of the hidden $S U(d)$ group of symmetries for a system on a $d$ sphere, and spawned extensive literature on algebras of conserved quantities of the Higgs oscillator. The energy spectrum of the Higgs oscillator is of the form (2).

We now define, with some geometric preliminaries, the Higgs oscillator Hamiltonian. Consider a unit $d$-sphere embedded in a $(d+1)$-dimensional flat space as

$$
x_{0}^{2}+x_{1}^{2}+\cdots+x_{d}^{2}=1
$$

and parametrized by the angles $\theta_{1}, \ldots, \theta_{d}$ as

$$
\begin{aligned}
x_{d} & =\cos \theta_{d}, \\
x_{d-1} & =\sin \theta_{d} \cos \theta_{d-1}, \\
x_{1} & =\sin \theta_{d} \ldots \sin \theta_{2} \cos \theta_{1}, \\
x_{0} & =\sin \theta_{d} \ldots \sin \theta_{2} \sin \theta_{1} .
\end{aligned}
$$

The sphere is endowed with the standard round metric defined recursively in $d$,

$$
d s_{S^{d}}^{2}=d \theta_{d}^{2}+\sin ^{2} \theta_{d} d s_{S^{d-1}}^{2}, \quad d s_{S^{1}}^{2}=d \theta_{1}^{2} .
$$

Similarly, the corresponding Laplacian is defined recursively,

$$
\begin{aligned}
\Delta_{S^{d}} & =\frac{1}{\sin ^{d-1} \theta_{d}} \partial_{\theta_{d}}\left(\sin ^{d-1} \theta_{d} \partial_{\theta_{d}}\right)+\frac{1}{\sin ^{2} \theta_{d}} \Delta_{S^{d-1}}, \\
\Delta_{S^{1}} & =\partial_{\theta_{1}}^{2} .
\end{aligned}
$$

The Higgs oscillator is a particle on a $d$-sphere moving in a potential varying as the inverse cosine squared of the polar angle, 


$$
H=-\Delta_{S^{d}}+\frac{\alpha(\alpha-1)}{\cos ^{2} \theta_{d}}
$$

The energy spectrum is given by

$$
E_{N}=\left(N+\alpha+\frac{d-1}{2}\right)^{2}-\frac{(d-1)^{2}}{4},
$$

where $N$ is the energy level number. This expression is manifestly of the form (2).

To implement our geometrization program for the Higgs oscillator, one can work directly with (12), which takes the form

$$
\begin{aligned}
& \frac{d-1}{2} \frac{\Omega}{\sin ^{d-1} \theta_{d}} \partial_{\theta_{d}}\left(\sin ^{d-1} \theta_{d} \partial_{\theta_{d}} \Omega\right)-\frac{d^{2}-1}{4}\left(\partial_{\theta_{d}} \Omega\right)^{2} \\
& +\left(C+\frac{\alpha(\alpha-1)}{\cos ^{2} \theta_{d}}\right) \Omega^{2}=m^{2} .
\end{aligned}
$$

Substituting $\Omega=\cos \theta_{d}$ produces only two constraints on the parameters to ensure that the equation is satisfied,

$$
C=\frac{(d-1)^{2}}{4}, \quad m^{2}=\alpha(\alpha-1)+\frac{d^{2}-1}{4} .
$$

The value of $C$ above agrees with the one in (25), while the relation between the Klein-Gordon mass and the Higgs potential strength is the same as found in [7]. The output of our construction is thus a family of Klein-Gordon equations on the spacetime

$$
d s^{2}=\frac{-d t^{2}+d s_{S^{d}}^{2}}{\cos ^{2} \theta_{d}},
$$

which is precisely the (global) anti-de Sitter spacetime $\operatorname{AdS}_{d+1}$. We note that rational values of $\alpha$ in (24) correspond to Klein-Gordon masses in AdS for which the frequency spectrum (7) is perfectly resonant (all frequencies are integer in appropriate units) rather than merely strongly resonant (differences of any two frequencies are integer in appropriate units).

A remarkable property of the Higgs oscillator is that the metric (28) does not depend on the Higgs potential strength (which only affects the value of the Klein-Gordon mass). This feature is not replicated for more complicated potentials. Conversely, this implies that the AdS spacetime possesses a resonant spectrum of frequencies for fields of all masses (this statement can in fact be extended to fields of higher spins), rather than for fields of one specific mass. It is tempting to conjecture that AdS spacetime (being a maximally symmetric spacetime) is the only spacetime with this property, though we do not know a proof. Relations between Klein-Gordon equations of different masses have recently surfaced in the literature on "mass ladder operators" [28-31].

\section{ROSOCHATIUS}

\section{A. The superintegrable Rosochatius system}

The superintegrable Rosochatius system is the most direct generalization of the Higgs oscillator on a $d$-sphere preserving its superintegrability. General Rosochatius systems [19] were among the first Liouville-integrable systems discovered. A restriction on the potential makes these systems maximally superintegrable. The Higgs oscillator can be recovered by a further restriction of the potential as a particularly simple special case. Such systems are thus an ideal testing ground for applying our machinery, which has already been shown to work for the Higgs oscillator.

The superintegrable Rosochatius systems we deal with here are defined by the following family of Hamiltonians,

$$
H_{d}^{(R)}=-\Delta_{S^{d}}+\sum_{k=0}^{d} \frac{\alpha_{k}\left(\alpha_{k}-1\right)}{x_{k}^{2}} .
$$

The explicit form of the Laplacian and coordinates on the unit $d$-sphere can be read off from (21)-(23). The standard more general definition of the Rosochatius system $[19,20]$ additionally includes a harmonic potential with respect to the $x_{k}$ variables, $\sum_{k} \gamma_{k} x_{k}^{2}$, which gives an integrable system. If this harmonic potential is omitted, as we did above, the system becomes maximally superintegrable, as mentioned, for instance, in $[17,18]$.

In order to find the spectrum of the above Hamiltonian, we have to apply recursively the solution of the famed onedimensional Pöschl-Teller problem [24]. While this material is completely standard and occasionally covered in textbooks, we find the summary given in [32] concise and convenient. The energy eigenstates of the Pöschl-Teller Hamiltonian

$$
H_{P T}=-\partial_{x}^{2}+\frac{\mu(\mu-1)}{\cos ^{2} x}+\frac{\nu(\nu-1)}{\sin ^{2} x}
$$

are given by

$$
\varepsilon_{n}=(\mu+\nu+2 n)^{2}, \quad n=0,1,2, \ldots
$$

We do not need the explicit form of the eigenfunctions satisfying $H_{P T} \Psi_{n}=\varepsilon_{n} \Psi_{n}$ (though it is known).

Because of the recursion relations on $d$-spheres outlined above, the Rosochatius Hamiltonian (29) can likewise be defined recursively,

$$
\begin{aligned}
H_{d}^{(R)}= & -\frac{1}{\sin ^{d-1} \theta_{d}} \partial_{\theta_{d}}\left(\sin ^{d-1} \theta_{d} \partial_{\theta_{d}}\right)+\frac{\alpha_{d}\left(\alpha_{d}-1\right)}{\cos ^{2} \theta_{d}} \\
& +\frac{1}{\sin ^{2} \theta_{d}} H_{(d-1)}^{(R)}, \\
H_{1}^{(R)}= & -\partial_{\theta_{1}}^{2}+\frac{\alpha_{1}\left(\alpha_{1}-1\right)}{\cos ^{2} \theta_{1}}+\frac{\alpha_{0}\left(\alpha_{0}-1\right)}{\sin ^{2} \theta_{1}} .
\end{aligned}
$$

The variables separate, and if one substitutes the wave function in the form 


$$
\Psi\left(\theta_{1}, \ldots, \theta_{d}\right)=\prod_{p=1}^{d} \frac{\chi_{p}\left(\theta_{p}\right)}{\sin ^{(p-1) / 2} \theta_{p}},
$$

one obtains a recursive family of one-dimensional eigenvalue problems, all of which are of the Pöschl-Teller form,

$$
\begin{aligned}
& {\left[-\partial_{\theta_{d}}^{2}+\frac{\alpha_{d}\left(\alpha_{d}-1\right)}{\cos ^{2} \theta_{d}}+\left(\frac{(d-2)^{2}-1}{4}+E_{d-1}\right) \frac{1}{\sin ^{2} \theta_{d}}\right.} \\
& \left.-\frac{(d-1)^{2}}{4}\right] \chi_{d}=E_{d} \chi_{d} \\
& {\left[-\partial_{\theta_{1}}^{2}+\frac{\alpha_{1}\left(\alpha_{1}-1\right)}{\cos ^{2} \theta_{1}}+\frac{\alpha_{0}\left(\alpha_{0}-1\right)}{\sin ^{2} \theta_{d}}\right] \chi_{1}=E_{1} \chi_{1}}
\end{aligned}
$$

where $E_{d}$ are eigenvalues of $H_{d}^{(R)}$. Each subsequent equation introduces one new quantum number which we denote $n_{d}$.

The recursive solution of (35) proceeds as follows. First, the solution at $d=1$ is given by (31) as

$$
E_{1}\left(n_{1}\right)=\left(\alpha_{0}+\alpha_{1}+2 n_{1}\right)^{2}
$$

At $d=2$, one gets

$$
\begin{aligned}
& {\left[-\partial_{\theta_{2}}^{2}+\frac{\alpha_{2}\left(\alpha_{2}-1\right)}{\cos ^{2} \theta_{2}}\right.} \\
& \left.+\frac{\left(\alpha_{0}+\alpha_{1}+2 n_{1}+\frac{1}{2}\right)\left(\alpha_{0}+\alpha_{1}+2 n_{1}-\frac{1}{2}\right)}{\sin ^{2} \theta_{2}}-\frac{1}{4}\right] \chi_{2} \\
& =E_{2} \chi_{2} \text {. }
\end{aligned}
$$

Hence,

$E_{2}\left(n_{1}, n_{2}\right)=\left(\alpha_{0}+\alpha_{1}+\alpha_{2}+2 n_{1}+2 n_{2}+\frac{1}{2}\right)^{2}-\frac{1}{4}$.

The general pattern can now be guessed as

$$
\begin{aligned}
E_{d}\left(n_{1}, \ldots, n_{d}\right) \\
=\left(\alpha_{0}+\cdots+\alpha_{d}+2 n_{1}+\cdots+2 n_{d}+\frac{d-1}{2}\right)^{2} \\
\quad-\frac{(d-1)^{2}}{4} .
\end{aligned}
$$

It is straightforward to prove inductively that this expression persists under the recursion given by (35). Note that (39) is manifestly of the form (2). A classical version of the same construction, recursively expressing the superintegrable Rosochatius Hamiltonian through the action-angle variables has been given in [18].

\section{B. Klein-Gordonization of the superintegrable Rosochatius system}

To demonstrate how the geometrization procedure we have proposed above operates, we now apply it to the superintegrable Rosochatius system. For the purposes of demonstration, we use the simplest formulation outlined in Sec. II B, which allows one to utilize known explicit solutions for ground state wave functions to construct the relevant massless Klein-Gordon (wave) equation.

The only technical input we need is the form of the ground state wave function of the Pöschl-Teller Hamiltonian (30) given by

$$
\psi_{0}=\cos ^{\mu} x \sin ^{\nu} x .
$$

(This form satisfies the standard boundary conditions for physical wave functions only for $\mu \geq 0$ and $\nu \geq 0$. If not, $\mu$ must be replaced by $1-\mu$, and correspondingly for $\nu$. This is, however, completely irrelevant for our application of $\psi_{0}$ to construct geometrical conformal factors, and the above form, without any modifications, is perfectly suitable for our purposes.) From (40) and the recursive construction (34)-(39), one gets for the ground state wave function of the superintegrable Rosochatius Hamiltonian (29)

$\Psi_{0}\left(\theta_{1}, \ldots, \theta_{d}\right)=\prod_{p=1}^{d}\left[\left(\cos \theta_{p}\right)^{\alpha_{p}}\left(\sin \theta_{p}\right)^{\alpha_{0}+\alpha_{1}+\cdots+\alpha_{p-1}}\right]$.

On the other hand, $B$ defined by (2) can be read off (39) as

$$
B=\alpha_{0}+\alpha_{1}+\cdots+\alpha_{d}+\frac{d-1}{2} .
$$

We can hence directly apply the algorithm of Sec. II B by introducing

$$
\omega=\prod_{p=1}^{d}\left[\left(\cos \theta_{p}\right)^{\alpha_{p}}\left(\sin \theta_{p}\right)^{\alpha_{0}+\alpha_{1}+\cdots+\alpha_{p-1}}\right],
$$

under the assumption that

$$
\alpha_{0}+\alpha_{1}+\cdots+\alpha_{d}+\frac{d-1}{2}=0 .
$$

This yields a $d$-parameter family of spacetimes given by (16) whose massless wave equations possess perfectly resonant spectra and geometrize the superintegrable Rosochatius problem,

$$
d s^{2}=\omega^{\frac{4}{d-1}}\left(-d t^{2}+d s_{S^{d}}^{2}\right) .
$$

[Note that setting $\alpha_{d}=-(d-1) / 2$ and the rest of $\alpha_{p}$ to 0 returns the case of Higgs oscillator with the coupling 
strength corresponding to zero mass in the Klein-Gordon equation, while (45) becomes the AdS metric.]

For a final statement of our result, it is convenient to reparametrize $\alpha_{p}$ as

$$
\begin{aligned}
& \alpha_{p}=-\frac{d-1}{2} \beta_{p} \text { for } p \geq 1, \\
& \alpha_{0}=-\frac{d-1}{2}\left(1-\beta_{1}-\cdots-\beta_{d}\right) .
\end{aligned}
$$

In terms of $\beta_{p}$, (45) becomes

$$
d s^{2}=\frac{-d t^{2}+d s_{S^{d}}^{2}}{\prod_{p=1}^{d}\left[\left(\cos \theta_{p}\right)^{2 \beta_{p}}\left(\sin \theta_{p}\right)^{2\left(1-\beta_{p}-\cdots-\beta_{d}\right)}\right]} .
$$

This evidently agrees with (28) when $\beta_{d}=1$ and the rest of $\beta_{p}$ are 0 .

\section{OUTLOOK}

We have presented a procedure (Klein-Gordonization) associating to quantum systems of the form (1) a KleinGordon equation on a static spacetime given by (16). For systems with the quadratic energy spectrum (2), our procedure results in spacetimes with a resonant spectrum of evenly spaced frequencies (7). This correspondence generalizes the previously known relation between the Higgs oscillator (24) and (global) anti-de Sitter spacetime (28).

Implementing our procedure in practice requires solving a nonlinear elliptic equation, which can be written as (12) or (15). The latter form is closely reminiscent of elliptic equations extensively studied in relation to classic prescribed scalar curvature problems of differential geometry (though the exact power appearing in the power-law nonlinearity is different). If one aims at constructing a massless Klein-Gordon (i.e., wave) equation corresponding to the original quantum-mechanical system, the nonlinearity drops out, resulting in a much simpler problem. In this case, known ground state wave functions for the original quantum system can be utilized for the conversion procedure, as described in Sec. II B. We have demonstrated how this approach works for superintegrable Rosochatius systems (29), resulting in a family of spacetimes (47) resonant with respect to the massless wave equation.

We conclude with a list of open questions relevant for our formalism.

(i) General theory of existence of solutions of (15) would contribute appreciably to clarifying the operation of our formalism. Similar equations arising in differential geometry [6] have been thoroughly analyzed; hence one should expect that the situation for our equation may as well be elucidated.

(ii) In practical applications of our formalism, we have focused on the case of zero Klein-Gordon mass, where (15) greatly simplifies. Are there any general techniques for solving this equation (rather than analyzing the existence of solutions) for nonzero masses (at least, for solvable potentials in the original quantum-mechanical system)?

(iii) Equation (15) may, in principle, admit multiple solutions, given that there is freedom in choosing boundary conditions, depending on which conformal transformation one allows. Singular conformal transformations may also be allowed (and they may push boundaries at finite distance off to infinity). This is in fact the case for the AdS construction starting from the Higgs oscillator. It would be good to quantify this freedom in choosing solutions of (15) and understand which prescriptions result in spacetimes interesting from a physical perspective.

(iv) Systems with quadratic spectra exist in extensions of the class of Hamiltonians we have considered here, given by (1). For example, it is possible to include effects of monopole fields without distorting the spectrum [33]. Klein-Gordonization is likely to generalize to such systems, resulting in KleinGordon equations with background gauge fields.

(v) It would be interesting to understand how the spacetimes resulting from our construction, such as (47), function in the context of dynamical theories of gravity. For instance, anti-de Sitter spacetime solves Einstein's equations with a negative cosmological constant. More complicated spacetimes may require some matter fields to be supported as solutions. In the context of dynamical theories, the resonant linear spectra of our spacetimes guarantee that weakly nonlinear dynamics of their perturbations is highly sophisticated. (Nonlinear instability of AdS, which is precisely a manifestation of such phenomena, is a broad currently active research area.)

(vi) What are the symmetry properties of spacetimes generated by Klein-Gordonization? How do they connect to the symmetries of the original quantummechanical problem (and, in particular, hidden symmetries)? Again, for the case of the Higgs oscillator, this perspective has turned out to be fruitful, and it would be good to see how it works in more general cases.

\section{ACKNOWLEDGMENTS}

The work of O. E. is funded under a CUniverse research promotion project by Chulalongkorn University (Grant No. CUAASC). O.E. furthermore thanks Marian Smoluchowski Institute of Physics in Krakow and support from Polish National Science Centre Grant No. DEC-2012/ 06/A/ST2/00397, as well as Instituto de Fisica Teorica (IFT UAM-CSIC) in Madrid for its support via the Centro de Excelencia Severo Ochoa Program under Grant No. SEV2016-0597 during collaboration visits while this work was 
in progress, and specifically Piotr Bizoń and Antonio González-Arroyo for hospitality and discussions. The work of H. D. and A. N. was partially supported by the Armenian State Committee of Science Grant No. 15T-1C367 and was done within the ICTP programs NT04 and AF04. We furthermore thank the anonymous referee for providing an extremely detailed report with a number of stimulating suggestions.
[1] B. Rink, Lecture notes on geometric mechanics and dynamics, http://www.few.vu.nl/ brink/Preview.pdf.

[2] G. W. Gibbons, The Jacobi metric for timelike geodesics in static spacetimes, Classical Quantum Gravity 33, 025004 (2016).

[3] S. Chanda, G. W. Gibbons, and P. Guha, Jacobi-MaupertuisEisenhart metric and geodesic flows, J. Math. Phys. 58, 032503 (2017).

[4] H. Yamabe, On a deformation of Riemannian structures on compact manifolds, Osaka Journal of mathematics 12, 21 (1960).

[5] J. M. Lee and T. H. Parker, The Yamabe problem, Bull. Am. Math. Soc. 17, 37 (1987).

[6] T. Aubin, Some Nonlinear Problems in Riemannian Geometry (Springer, New York, 1998).

[7] O. Evnin and C. Krishnan, A hidden symmetry of AdS resonances, Phys. Rev. D 91, 126010 (2015).

[8] O. Evnin and R. Nivesvivat, AdS perturbations, isometries, selection rules, and the Higgs oscillator, J. High Energy Phys. 01 (2016) 151.

[9] O. Evnin and R. Nivesvivat, Hidden symmetries of the Higgs oscillator and the conformal algebra, J. Phys. A 50, 015202 (2017).

[10] P. W. Higgs, Dynamical symmetries in a spherical geometry 1, J. Phys. A 12, 309 (1979).

[11] H. I. Leemon, Dynamical symmetries in a spherical geometry 2, J. Phys. A 12, 489 (1979).

[12] B. Craps, O. Evnin, and J. Vanhoof, Renormalization group, secular term resummation, and AdS (in)stability, J. High Energy Phys. 10 (2014) 48.

[13] B. Craps, O. Evnin, and J. Vanhoof, Renormalization, averaging, conservation laws and AdS (in)stability, J. High Energy Phys. 01 (2015) 108.

[14] I.-S. Yang, Missing top of the AdS resonance structure, Phys. Rev. D 91, 065011 (2015).

[15] P. Bizoń and A. Rostworowski, On Weakly Turbulent Instability of Anti-de Sitter Space, Phys. Rev. Lett. 107, 031102 (2011).

[16] B. Craps and O. Evnin, AdS (in)stability: An analytic approach, Fortsch. Phys. 64, 336 (2016).

[17] J. Harnad and O. Yermolayeva, Superintegrability, Lax matrices, and separation of variables, CRM Proc. Lect. Notes 37, 65 (2004).
[18] A. Galajinsky, A. Nersessian, and A. Saghatelian, Superintegrable models related to near horizon extremal MyersPerry black hole in arbitrary dimension, J. High Energy Phys. 06 (2013) 002.

[19] E. Rosochatius, Doctoral dissertation, University of Göttingen, 1877.

[20] Encyclopedia of Integrable Systems, edited by A. B. Shabat et al., http://home.itp.ac.ru/ adler/E/e.pdf.

[21] M. Feigin, O. Lechtenfeld, and A. P. Polychronakos, The quantum angular Calogero-Moser model, J. High Energy Phys. 07 (2013) 162.

[22] T. Hakobyan, O. Lechtenfeld, and A. Nersessian, Superintegrability of generalized Calogero models with oscillator or Coulomb potential, Phys. Rev. D 90, 101701 (2014).

[23] F. Correa, T. Hakobyan, O. Lechtenfeld, and A. Nersessian, Spherical Calogero model with oscillator/Coulomb potential: Quantum case, Phys. Rev. D 93, 125009 (2016).

[24] G. Pöschl and E. Teller, Bemerkungen zur Quantenmechanik des anharmonischen Oszillators, Zeitschr. Phys. 83, 143 (1933).

[25] N. D. Birrell and P. C. W. Davies, Quantum Field Theory in Curved Spacetime (CUP, Cambridge, 1986).

[26] F. Correa, V. Jakubsky, and M. S. Plyushchay, AharonovBohm effect on $\operatorname{AdS}(2)$ and nonlinear supersymmetry of reflectionless Poschl-Teller system, Ann. Phys. (Amsterdam) 324, 1078 (2009).

[27] M. Lakshmanan and K. Eswaran, Quantum dynamics of a solvable nonlinear chiral model, J. Phys. A 8, 1658 (1975).

[28] V. Cardoso, T. Houri, and M. Kimura, Mass ladder operators from spacetime conformal symmetry, Phys. Rev. D 96, 024044 (2017).

[29] D. Karateev, P. Kravchuk, and D. Simmons-Duffin, Weight shifting operators and conformal blocks, arXiv:1706.07813.

[30] V. Cardoso, T. Houri, and M. Kimura, General first-order mass ladder operators for Klein-Gordon fields, arXiv: 1707.08534.

[31] W. Mück, Ladder operators for Klein-Gordon equation with scalar curvature term, arXiv:1710.01283.

[32] L. Infeld and T.E. Hull, The factorization method, Rev. Mod. Phys. 23, 21 (1951).

[33] L. Mardoyan, A. Nersessian, and A. Yeranyan, Relationship between quantum mechanics with and without monopoles, Phys. Lett. A 366, 30 (2007). 